\title{
Moritz Kerz’ Beiträge zur modernen Klassenkörpertheorie
}

\author{
Marc Levine
}

\begin{abstract}
Moritz Kerz an der Universität Regensburg wurde die erste Minkowski-Medaille der DMV verliehen in Anerkennung seiner herausragenden Beiträge zur arithmetischen algebraischen Geometrie und algebraischen $K$-Theorie. Kerz befasst sich mit zentralen Fragen zu algebraischen Zykeln, höherer algebraischer K-Theorie, höherer Klassenkörpertheorie arithmetischer Schemata und den Verbindungen zwischen diesen Gebieten. Seine international bekannte Arbeit hat zu großen Fortschritten auf jedem dieser Gebiete geführt. Wir geben hier einen

Überblick auf einige seiner Resultate, wobei wir uns auf seine Beiträge zur höheren Klassenkörpertheorie arithmetischer Schemata beschränken.
\end{abstract}

\section{Galoistheoretischer Hintergund}

Sei $F$ ein Körper mit separablem Abschluss $\bar{F}$ (oder algebraischem Abschluss, falls char $(F)=0$ ). Das primäre Ziel der Galoistheorie seit ihrer Erfindung 1832 durch Évariste Galois ist es, den Körper $F$ durch die Gruppe derjenigen Automorphismen von $\bar{F}$, die auf $F$ als die Identität operieren, zu verstehen. Diese absolute Galoisgruppe $\mathrm{Gal}(\bar{F} / F)$ ist in überraschend wenigen Fällen tatsächlich bekannt. Für $F=\mathbb{C}((t))$, den Körper formaler Laurentreihen über $\mathbb{C}$, erhält man $\bar{F}$ durch Adjunktion aller $n$-ten Wurzeln $t^{1 / n}$ von $t$. Die Galoisgruppe $\operatorname{Gal}\left(F\left(t^{1 / n}\right) / F\right)$ ist $\mathbb{Z} / n \mathbb{Z}$, wobei 1 durch $t^{1 / n} \mapsto e^{2 \pi \cdot i / n} t^{1 / n}$ operiert. Fügt man all diese Galoigruppen zusammen, sieht man, dass $\operatorname{Gal}(\bar{F} / F)$ durch die Vervollständigung $\hat{\mathbb{Z}}$ von $\mathbb{Z}$ bezüglich aller nichtverschwindenden Untergruppen gegeben ist:

$$
\hat{\mathbb{Z}}=\lim _{\leftarrow} \mathbb{Z} / n \mathbb{Z} \cong \prod_{p} \mathbb{Z}_{p}
$$

Hier bezeichnet $\mathbb{Z}_{p}$ die $p$-adischen Zahlen und das Produkt ist als über alle Primzahlen $p$ laufend $z u$ verstehen. Dieses Resultat sollte an die Fundamentalgruppe von $S^{1}$, oder besser die Fundamentalgruppe der punktierten Einheitsscheibe $D^{0}$ in $\mathbb{C}$, erinnern; die eindeutige zusammenhängende Überlagerung von $D^{0}$ von Grad $n$ erhält man als die zur $n$-ten Wurzel des Parameters $z$ gehörige Riemannsche Fläche, wobei $1 \in \mathbb{Z}=\pi_{1}\left(D^{0}, 1 / 2\right)$ genau wie oben durch $z^{1 / n} \mapsto e^{2 \pi \cdot i / n} z^{1 / n}$ operiert. Dieses Beispiel illustriert die Ähnlichkeiten zwischen Galoistheorie und der Theorie von Überlagerungen und der Fundamentalgruppe. Der grundlegende Unterschied ist, dass Galoistheorie aus endlichen Erweiterungen entsteht, während in der Topologie auch Überlagerungen unendlichen Grades betrachtet werden. Dieser Unterschied führt zum Übergang von $\pi_{1}\left(D^{0}, 1 / 2\right)=\mathbb{Z}$ zur Vervollständigung $\operatorname{Gal}(\overline{\mathbb{C}((t))} / \mathbb{C}((t)))=\hat{\mathbb{Z}}$.

Für einen endlichen Körper $\mathbb{F}_{q}$ mit $q$ Elementen, $q=p^{n}$ eine Primpotenz, mit algebraischem Abschluss $\overline{\mathbb{F}}$, ist die absolute Galoigruppe $\mathrm{Gal}\left(\overline{\mathbb{F}}_{q} / \mathbb{F}_{q}\right)$ auch isomorph zu $\hat{\mathbb{Z}}$; im Wesentlichen weil $\mathbb{F}_{q}$ genau eine Erweiterung vom Grad $n$ hat, nämlich $\mathbb{F}_{q^{n}}$. Hier gibt es einen wichtigen Unterschied:
$\operatorname{Gal}\left(\overline{\mathbb{F}}_{q} / \mathbb{F}_{q}\right)$ enthält einen kanonischen topologischen Erzeuger, den Frobeniusautomorphismus $\operatorname{Frob}_{q}(x)=x^{q}$. Das bedeutet, dass für jedes $n$ die Einschränkung von Frob $_{q}$ zu einem Automorphismus von $\mathbb{F}_{q^{n}}$ über $\mathbb{F}_{q}$ die Galoisgruppe $\operatorname{Gal}\left(\mathbb{F}_{q^{n}} / \mathbb{F}_{q}\right)$ erzeugt.

Für die meisten natürlich vorkommenden Körper $F$ bleibt die absolute Galoisgruppe ein Mysterium. Selbst für $F=\mathbb{Q}$ ist sie unbekannt. Die noch unbewiesene Shafarevichvermutung besagt, dass $\mathrm{Gal}\left(\overline{\mathbb{Q}} / \mathbb{Q}^{\mathrm{ab}}\right)$ eine freie (profinite) Gruppe von abzählbarem Rang ist. Hier bezeichnet $\mathbb{Q}^{\mathrm{ab}} \subset \overline{\mathbb{Q}}$ die maximale abelsche Erweiterung von $\mathbb{Q}$, das heißt die Vereinigung aller derjenigen endlichen Erweiterungen $\mathbb{Q} \subset K$, für die $\mathrm{Gal}(K / \mathbb{Q})$ abelsch ist. Nach dem Satz von KroneckerWeber sind $\mathbb{Q}^{\mathrm{ab}}$ und die Galoisgruppe $\operatorname{Gal}\left(\mathbb{Q}^{\mathrm{ab}} / \mathbb{Q}\right)$ bekannt.

Satz 1 (Kronecker-Weber. Erster vollständiger Beweis durch Hilbert (1896)). Die maximale abelsche Erweiterung $\mathbb{Q}^{\mathrm{ab}}$ ist als Unterkörper von $\mathbb{C}$ über $\mathbb{Q}$ durch die Einheitswurzeln erzeugt: $\mathbb{Q}^{\mathrm{ab}}=\mathbb{Q}\left(\left\{e^{2 \pi \cdot i / n} \mid n \in \mathbb{N}\right\}\right)$. Die Galoisgruppe $\operatorname{Gal}\left(\mathbb{Q}^{\mathrm{ab}} / \mathbb{Q}\right)$ ist $\hat{\mathbb{Z}}$.

Ein Verwandter der Shafarevichvermutung ist das inverse Galoisproblem, ebenfalls ungelöst. Es besagt, dass jede endliche Gruppe als Quotient von Gal $(\overline{\mathbb{Q}} / \mathbb{Q})$ auftritt. Mit anderen Worten, für jede endliche Gruppe $G$, soll eine endliche Erweiterung $\mathbb{Q} \subset E$ mit $G \cong \operatorname{Gal}(E / \mathbb{Q})$ existieren. Die Shafarevichvermutung impliziert eine positive Antwort auf das inverse Galoisproblem über $\mathbb{Q}^{\mathrm{ab}}$.

Für $F$ ein Körper von Transzendenzgrad 1 über einem endlichen Körper $\mathbb{F}_{q}$, wurde das Analogon der Shafarevichvermutung von Harbater [19, Cor. 4.2] und Pop [30] bewiesen, im Wesentlichen durch die Lösung einer schwachen Version des inversen Galoisproblems über $F^{\mathrm{ab}}$.

2

Geometrische Zugänge zur Klassenkörpertheorie: der klassische Fall

Im Gegensatz zu unserem schlechten Verständnis der absoluten Galoisgruppe ist relativ viel über $\operatorname{Gal}\left(F^{\mathrm{ab}} / F\right)$ bekannt; es ist der maximale abelsche Quotient von $\operatorname{Gal}(\bar{F} / F)$. Der klassische Fall behandelt einen globalen Körper $k$. Solch 
ein Körper ist entweder ein Zahlkörper (eine endliche Erweiterung von $\mathbb{Q}$ ) oder ein Körper von Transzendenzgrad 1 über einem endlichen Körper. In letzterem Fall ist $k$ eine endliche Erweiterung von $\mathbb{F}_{p}(t)$, allerdings ist im Kontrast zu Zahlkörpern die Erweiterung $\mathbb{F}_{p}(t) \subset k$ nicht eindeutig definiert.

Der Satz von Kronecker-Weber ermöglicht eine explizite Beschreibung von $\mathbb{Q}^{\mathrm{ab}}$ und der Galoisgruppe $\operatorname{Gal}\left(\mathbb{Q}^{\mathrm{ab}} / \mathbb{Q}\right)$ und man erkennt, dass $\mathrm{Gal}\left(\mathbb{Q}^{\mathrm{ab}} / \mathbb{Q}\right)=\hat{\mathbb{Z}}$ recht direkt aus den ganzen Zahlen $\mathbb{Z} \subset \mathbb{Q}$ und ihren Idealen konstruiert werden kann. Davon ausgehend, liefert in ihrer klassischen Form die Klassenkörpertheorie eine Beschreibung der abelianisierten Galoisgruppe eines globalen Körpers $k$ durch Objekte „innerhalb $k^{\prime \prime}$. Am einfachsten ist der unverzweigte Fall zu beschreiben.

Satz 2 (Furtwängler (1906)). Sei k ein Zahlkörper und $k_{\mathrm{Hilb}}$ der Hilbertsche Klassenkörper von $k$, nämlich die maximale abelsche unverzweigte Erweiterung von $k$. Dann definiert ein Primideal $P$ in $\mathcal{O}_{k}$ auf den zugehörigen Frobeniusautomorphismus von $k_{\text {Hilb }}$ abzubilden einen Isomorphismus zwischen der Klassengruppe $\mathrm{Cl}(k)$ von $k$ und der Galoisgruppe $\mathrm{Gal}\left(k_{\mathrm{Hilb}} / k\right)$.

Einige Erläuterungen: $\mathcal{O}_{k} \subset k$ bezeichnet den Ganzheitsring von $k$, nämlich den Unterring von $k$ der alle Elemente aus $k$ enthält, die Nullstellen eines normierten Polynoms mit Koeffizienten in $\mathbb{Z}$ sind. In $\mathcal{O}_{k}$ zerfällt jedes nichtverschwindende Ideal eindeutig in ein Produkt von Primidealen. Eine Erweiterung $k \subset k^{\prime}$ heißt unverzweigt über einem Primideal $P \subset \mathcal{O}_{k}$, falls das Ideal $P \mathcal{O}_{k^{\prime}}$ in ein Produkt $P \mathcal{O}_{k^{\prime}}=\prod_{i=1}^{r} Q_{i}$ verschiedener Primideale $Q_{i}$ zerfällt. Die Erweiterung ist unverzweigt, falls sie über jedem Primideal $P$ unverzweigt ist.

Ein gebrochenes Ideal in $k$ ist ein endlich erzeugter $\mathcal{O}_{k-}$ Untermodul von $k$. Die Menge aller gebrochenen Ideale bildet eine Gruppe unter Multiplikation von Untermoduln, und diese Gruppe ist erzeugt vom Untermonoid bestehend aus nichtverschwindenden Idealen in $\mathcal{O}_{k}$. Ein gebrochenes Hauptideal ist ein gebrochenes Ideal der Form $\mathcal{O}_{k} \cdot f$ für ein $f \in k \backslash\{0\}$ und die Klassengruppe $\mathrm{Cl}(k)$ ist die Quotientengruppe aller gebrochenen Ideale modulo der gebrochenen Hauptideale. Die Tatsache, dass jedes Ideal in $\mathcal{O}_{k}$ eindeutig in ein Produkt von Primidealen zerfällt, bedeutet, dass die Gruppe der gebrochenen Ideale die freie abelsche Gruppe auf der Mengen der nichtverschwindenden Primideale in $\mathcal{O}_{k}$ ist. Wir bezeichnen Letztere mit $Z_{0}\left(\mathcal{O}_{k}\right)$. Ein wichtiges Resultat in dieser Richtung ist die Endlichkeit der Klassengruppe; ihr Beweis lässt sich zu Minkowskis Gitterpunktsatz über konvexe Mengen zurückverfolgen.

Um $Z_{0}\left(\mathcal{O}_{k}\right)$ in $\operatorname{Gal}\left(k_{\mathrm{Hilb}} / k\right)$ abzubilden benutzen wir die Frobeniusautomorphismen von endlichen Körpern. Sei $P \subset \mathcal{O}_{k}$ ein nichtverschwindendes Primideal und $Q \subset \mathcal{O}_{k_{\text {Hilb }}}$ ein Primideal über $P$. Die Inklusion $\mathcal{O}_{k} \subset \mathcal{O}_{k_{\text {Hilb }}}$ induziert die Erweiterung $\kappa(P):=\mathcal{O}_{k} / P \subset \kappa(Q):=\mathcal{O}_{k_{\mathrm{Hilb}}} / Q$ zwischen den endlichen Restklassenkörper von $P$ und $Q$. Ist $\kappa(P)=\mathbb{F}_{q}$, so haben wir den Frobeniusautomorphismus $\operatorname{Frob}_{q}$ von $\kappa(Q)$ über $\kappa(P)$. Da $k_{\text {Hilb }}$ unverzweigt ist, existiert ein eindeutig bestimmtes Element $\operatorname{Frob}_{P} \in \operatorname{Gal}\left(k_{\mathrm{Hilb}} / k\right)$, das $Q$ fix hält und sich modulo $P$ zu Frob $q$ reduziert. Da Gal $\left(k_{\mathrm{Hilb}} / k\right)$ abelsch ist, hängt Frob $_{P}$ nur von $P$ und nicht von der Wahl von $Q$ ab und wir können die Reziprozitätsabbildung rec $_{k}: Z_{0}\left(\mathcal{O}_{k}\right) \rightarrow \operatorname{Gal}\left(k_{\mathrm{Hilb}} / k\right)$ definieren. Sie bildet eine Summe $\sum_{i} n_{i} P_{i}$ auf $\sum_{i} n_{i} \operatorname{Frob}_{P_{i}}$ ab. Die Reziprozitätsabbildung ist surjektiv und (was erheblich schwieriger zu zeigen ist) ihr Kern ist genau die Untergruppe der gebrochenen Hauptideale.

Wenn wir auf den Satz von Kronecker-Weber zurückblicken und ihn mit Furtwänglers Satz über $\mathbb{Q}$ vergleichen, sehen wir dass $\mathrm{Cl}(\mathbb{Q})=\{0\}$, denn jedes Ideal in $\mathbb{Z}$ ist ein Hauptideal, und daher dass $\mathbb{Q}_{\text {Hilb }}=\mathbb{Q}$. Diese Erkenntnis spiegelt sich im klassischen Resultat, dass jede nichttriviale endliche Erweiterung von $\mathbb{Q}$ verzweigt ist, wieder. Diese Tatsache folgt auch aus Minkowskis Gitterpunktsatz.

Im geometrischen Fall eines Körpers $k$ mit Transzendenzgrad 1 über einem endlichen Körper $\mathbb{F}_{q}$ nehmen wir an, dass $q$ maximal bezüglich $k$ gewählt ist, das heißt, ein Element von $k$, das algebraisch über $\mathbb{F}_{q}$ ist, liegt bereits in $\mathbb{F}_{q}$. Dann ist $k$ der Körper rationaler Funktionen auf einer eindeutig bestimmten glatten eigentlichen ${ }^{1}$ algebraischen Kurve $C$ über $\mathbb{F}_{q}$, wobei $\mathbb{F}_{q}$ der Körper aller global definierten regulären Funktionen auf $C$ ist. Die Kurve $C$ heißt dann geometrisch irreduzibel über $\mathbb{F}_{q}$. Die Gruppe der gebrochenen Ideale wird ersetzt durch die Gruppe $Z_{0}(C)$ der Divisoren auf $C$, nämlich die freie abelsche Gruppe auf den (abgeschlossenen) Punkten von $C$. In der Tat sind im Fall eines Zahlkörpers $k$ die abgeschlossenen Punkte des Schemas Spec $\mathcal{O}_{k}$ genau die nichtverschwindenden Primideale in $\mathcal{O}_{k}$. Man hat ähnlich zur Situation eines Zahlkörpers eine Definition von "unverzweigt“. Ist $\overline{\mathbb{F}}$ der algebraische Abschluss von $\mathbb{F}_{q}$, so ist das Kompositum $\overline{\mathbb{F}} k$ eine abelsche unverzweigte Erweiterung von $k$ und ist deshalb ein Unterkörper der maximalen abelschen unverzweigten Erweiterung $k_{\mathrm{nr}}^{\mathrm{ab}}$ von $k$. Man erhält so eine exakte Sequenz

$$
0 \rightarrow \operatorname{Gal}\left(k_{\mathrm{nr}}^{\mathrm{ab}} / \overline{\mathbb{F}} k\right) \rightarrow \operatorname{Gal}\left(k_{\mathrm{nr}}^{\mathrm{ab}} / k\right) \rightarrow \operatorname{Gal}\left(\overline{\mathbb{F}} / \mathbb{F}_{q}\right) \cong \hat{\mathbb{Z}} \rightarrow 0
$$

zwischen (abelschen) Galoisgruppen.

Für jeden abgeschlossenen Punkt $x \in C$ hat man den Restklassenkörper $\kappa(x)$. Er ist eine endliche Erweiterung von $\mathbb{F}_{q}$. Die Abbildung $x \mapsto \operatorname{dim}_{\mathbb{F}_{q}}(\kappa(x))$ lässt sich $\mathbb{Z}$-linear zu einem Homomorphismus $\operatorname{deg}_{\mathbb{F}_{q}}: Z_{0}(C) \rightarrow \mathbb{Z}$ fortsetzen. Genau wie für Riemannsche Flächen, gegeben eine rationale Funktion $f$ auf $C$, erhält man den zugehörigen Hauptdivisor $\operatorname{div}(f) \in Z_{0}(C)$, nämlich den Divisor der Nullstellen minus der Pole von $f$ mit passenden Vielfachheiten, und $\operatorname{deg}_{\mathbb{F}_{q}} \operatorname{div}(f)=0$. Man definiert $\mathrm{CH}_{0}(C)$ als den Quotient $\mathrm{CH}_{0}(C):=Z_{0}(C) /\{\operatorname{div}(f) \mid f\}$ aller Divisoren auf $C$ modulo der Hauptdivisoren und erhält die Gradabbildung $\operatorname{deg}_{\mathbb{F}_{q}}: \mathrm{CH}_{0}(C) \rightarrow \mathbb{Z}$. Dem Satz von Furtwängler entspricht dann folgendes Resultat (siehe [32] für historische Details).

Satz 3 (Schmidt (1931) und Witt (1935)). Einen abgeschlossenen Punkt $x \in C$ auf den Frobeniusautomorphismus Frob $_{x} \in$ $\mathrm{Gal}\left(k_{\mathrm{nr}}^{\mathrm{ab}} / k\right)$ abzubilden definiert einen Isomorphismus zwischen $\mathrm{CH}_{0}(C)^{0}$ und $\mathrm{Gal}\left(k_{\mathrm{nr}}^{\mathrm{ab}} / \overline{\mathbb{F}} k\right)$, wobei $\mathrm{CH}_{0}(C)^{0} \subset \mathrm{CH}_{0}(C)$ den Kern der Gradabbildung bezeichnet. Dieselbe Abbildung definiert eine exakte Sequenz

$$
0 \rightarrow \mathrm{CH}_{0}(C) \rightarrow \mathrm{Gal}\left(k_{\mathrm{nr}}^{\mathrm{ab}} / k\right) \rightarrow \hat{\mathbb{Z}} / \mathbb{Z} \rightarrow 0,
$$

das heißt, $\mathrm{Gal}\left(k_{\mathrm{nr}}^{\mathrm{ab}} / k\right)$ ist isomorph zur Vervollständigung von $\mathrm{CH}_{0}(C)$ bezüglich der Gradabbildung. 
Für eine endliche abelsche Galoiserweiterung $k \rightarrow K$ globaler Körper, diesmal möglicherweise verzweigt, ist die Galoisgruppe immer noch durch Frobeniusautomorphismen für die unverzweigten Primideale/Punkte erzeugt. Aber um die Relationen zwischen ihnen zu beschreiben führt man den Begriff eines Moduls ${ }^{2}$ ein. Im geometrischen Fall, anstatt die Divisoren beliebiger rationaler Funktionen zuzulassen, beschränkt man sich auf die Untergruppe derjenigen Funktionen $f$ für die $f-1$ zu ausreichend hoher Ordnung an endlich vielen Punkten in $C$ verschwindet, wobei die Verschwindungsordnung (grob) der zulässigen Verzweigung entspricht. Genauer, sei $m=\sum_{i=1}^{r} m_{i} \cdot p_{i}$ ein Modul, wobei die $p_{i}$ abgeschlossene Punkte in $C$ und die $m_{i}$ natürliche Zahlen bezeichnen. Ferner sei $R_{0}(C, \mathrm{~m}) \subset Z_{0}\left(C \backslash\left\{p_{1}, \ldots, p_{r}\right\}\right)$ die Untergruppe $\{\operatorname{div}(f) \mid f\} \cup\{0\}$, wobei $f \in k^{\times}$diejenigen rationalen Funktionen durchläuft, für die $f-1$ in $p_{i}$ mindestens zur Ordnung $m_{i}$ verschwindet. Wir setzen

$$
\mathrm{CH}_{0}(C, \mathrm{~m})=Z_{0}\left(C \backslash\left\{p_{1}, \ldots, p_{r}\right\}\right) / R_{0}(C, \mathrm{~m}) .
$$

Sei $\mathrm{CH}_{0}(C, \mathrm{~m})^{0} \subset \mathrm{CH}_{0}(C, \mathrm{~m})$ die Untergruppe bestehend aus den Restklassen mit Grad 0. Gegeben ein passendes Maß für die zulässige Verzweigung (das wir nicht genauer festlegen, siehe [36, VI, §2, no. 12] für Einzelheiten), hat man das folgende Resultat.

Satz 4 (Rosenlicht, Lang $[27,28,33,34,36])$. Sei $k_{\mathrm{m}}^{\mathrm{ab}} \supset k$ die maximale abelsche Erweiterung von $k=\mathbb{F}_{q}(C)$ mit Verzweigung beschränkt durch $m$. Dann definiert $x \in Z_{0}(C, m)$ auf den Frobeniusautomorphismus Frob ${ }_{x}: k_{\mathrm{m}}^{\mathrm{ab}} \rightarrow k_{\mathrm{m}}^{\mathrm{ab}}$ abzubilden einen Isomorphismus $\operatorname{rec}^{0}: \mathrm{CH}_{0}(C, \mathrm{~m})^{0} \rightarrow \mathrm{Gal}\left(k_{\mathrm{m}}^{\mathrm{ab}} / \overline{\mathbb{F}} k\right)$ und eine Injektion rec: $\mathrm{CH}_{0}(C, \mathrm{~m}) \hookrightarrow \mathrm{Gal}\left(k_{\mathrm{m}}^{\mathrm{ab}} / k\right)$ mit Kokern $\hat{\mathbb{Z}} / \mathbb{Z}$.

Der Frobeniusautomorphismus Frob ${ }_{x}: k_{\mathrm{m}}^{\mathrm{ab}} \rightarrow k_{\mathrm{m}}^{\mathrm{ab}}$ ist durch dieselbe Konstruktion wie im unverzweigten Fall definiert, denn für $x=\sum n_{i} x_{i} \in Z_{0}(C, \mathfrak{m})$ ist $k_{\mathfrak{m}}^{\mathrm{ab}}$ über jedem der $x_{i}$ unverzweigt.

Erhöht man die Verschwindungsordnung und fügt man immer mehr Punkte hinzu, erhält man eine Beschreibung der vollen absoluten Galoisgruppe von $k$ durch dieses System von wachsenden aber endlichen Quotienten, wieder nach Vervollständigung bezüglich des Grads. Die arithmetische Version dieser Resultate wurde viel früher bewiesen, in Arbeiten von Artin, aber wir betonen hier den geometrischen Fall.

\section{Höherdimensionale Klassenkörpertheorie}

Beginnend in den 1980er Jahren wurden immer allgemeinere Beschreibungen der abelianisierten Galoisgruppe von Funktionenkörpern von Varietäten über einem endlichen Körper oder einem Zahlkörper bekannt. Dafür ist es nützlich eine Erweiterung der klassischen Galoistheorie von Körpern für globalere Objekte zur Hand zu haben. Diese Erweiterung ist Grothendiecks étale Fundamentalgruppe $\pi_{1}^{\text {ét }}(X, x)$ eines Schemas $X$ mit geometrischem Basispunkt $x$. Sie ist eine algebraische Version der klassischen topologischen Fundamentalgruppe eines Raums $T$. Für $X$ das zu einem Körper $k$ gehörige Schema Speck ist ein geometrischer Basispunkt die Wahl eines separablen Abschlusses

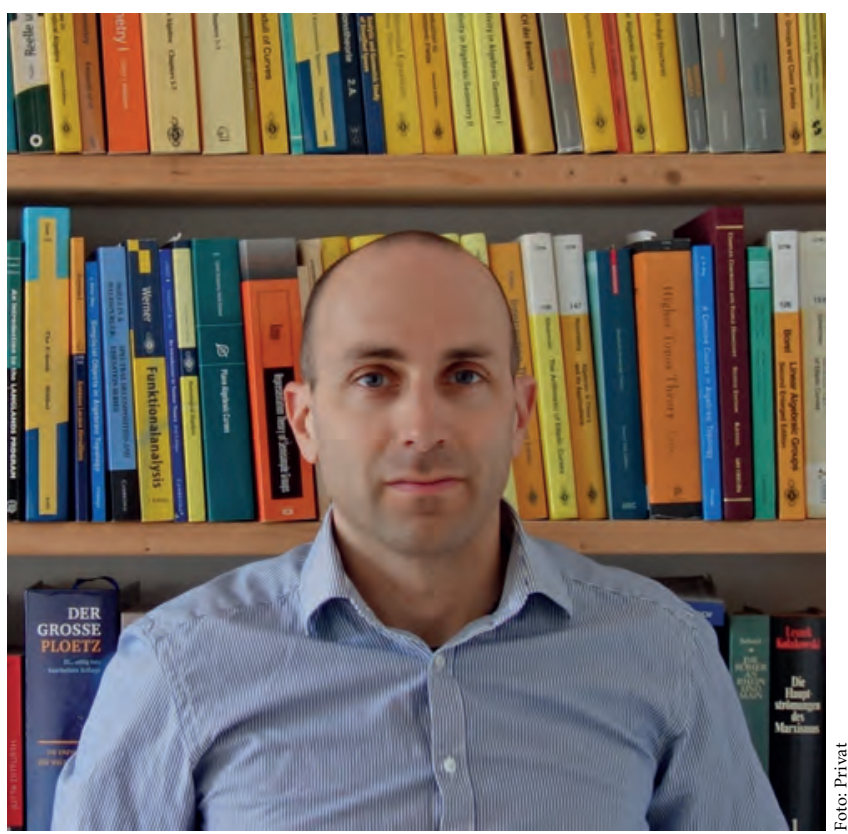

Moritz Kerz

$k \subset \bar{k}$ und $\pi_{1}^{\text {ét }}(X, x)$ ist die absolute Galoisgruppe $\operatorname{Gal}(\bar{k} / k)$. Ist $X$ ein Schema über $\mathbb{C}$ von endlichem Typ, das heißt, $X$ ist in endlichen vielen "Teilen“ durch Polynomgleichungen mit Koeffizienten aus $\mathbb{C}$ definiert, so ist ein geometrischer Basispunkt einfach nur ein gewöhnlicher Punkt von $X$ und, wenn wir zum topologischen Raum $X(\mathbb{C})$ mit der durch die Standardtopologie auf $\mathbb{C}$ induzierten Topologie übergehen, ist $\pi_{1}^{\text {ét }}(X, x)$ die Vervollständigung der topologischen Fundamentalgruppe $\pi_{1}(X(\mathbb{C}), x)$ bezüglich ihrer Untergruppen mit endlichem Index.

Für $X$ eine glatte und eigentliche Kurve über einem endlichen Körper $\mathbb{F}_{q}$ ist die Abelianisierung $\pi_{1}^{\text {ét }}(X, x)^{\mathrm{ab}}$ die Galoisgruppe der maximalen unverzweigten Erweiterung des Funktionenkörpers $\mathbb{F}_{q}(X)$, wie oben beschrieben. Ähnlicherweise ist für $\mathcal{O}_{k}$ der Ganzheitsring eines Zahlkörpers $k$ und $X=\operatorname{Spec} \mathcal{O}_{k}$ die Abelianisierung $\pi_{1}^{\text {et }}(X, x)^{\text {ab }}$ genau die Galoisgruppe über $k$ des Hilbertschen Klassenkörpers von $k$. Wegen der Abelianisierung wird die Wahl des Basispunkts irrelevant und wir schreiben deshalb einfach $\pi_{1}^{\mathrm{ab}}(X)$.

Für $X$ ein arithmetisches Schema ${ }^{3}$ hat jeder abgeschlossene Punkt $x$ in $X$ einen endlichen Restklassenkörper $\kappa(x)$. Man kann die obige Konstruktion von Frobeniuselementen in $\mathrm{Gal}(K / k)^{\mathrm{ab}}$ im Fall einer unverzweigten Erweiterung von globalen Körpern verallgemeinern und eine Abbildung $\operatorname{Gal}(\overline{\kappa(x)} / \kappa(x))=\pi_{1}^{\mathrm{ab}}(X) \rightarrow \pi_{1}^{\mathrm{ab}}(X)$ definieren. Die Gruppe $Z_{0}(X)$ von 0-Zykeln, das heißt, die freie abelsche Gruppe auf der Menge der abgeschlossenen Punkte in $X$, verallgemeinert gleichzeitig die Gruppe der gebrochenen Ideale im Fall eines Zahlenrings und die Divisorgruppe für eine Kurve über einem endlichen Körper, und unter der milden technischen Annahme, dass $X$ normal ist, definiert einen abgeschlossenen Punkt $x$ das Bild des Frobeniusautomorphismus unter der Abbildung $\pi_{1}^{\mathrm{ab}}(X) \rightarrow \pi_{1}^{\mathrm{ab}}(X)$ zuzuordnen eine Abbildung von $Z_{0}(X)$ nach $\pi_{1}^{\mathrm{ab}}(X)$ mit dichtem Bild. Das Hauptproblem ist, den Kern dieser Abbildung zu beschreiben. Um unsere Diskussion zu vereinfachen, kon- 
zentrieren wir uns auf den Fall von Varietäten über einem endlichen Körper.

Satz 5 (Kato-Saito (1983)). Sei X eine glatte, geometrisch irreduzible, projektive Varietät über einem endlichen Körper $\mathbb{F}_{q}$. Dann definieren die Frobeniusabbildungen auf abgeschlossenen Punkten in X einen injektiven Homomorphismus rec: $\mathrm{CH}_{0}(X) \rightarrow \pi_{1}^{\mathrm{ab}}(X)$, eine Reziprozitätsabbildung, mit Kokern $\hat{\mathbb{Z}} / \mathbb{Z}$, und einen Isomorphismus $\mathrm{CH}_{0}(X)^{0} \rightarrow \pi_{1}^{\mathrm{ab}}(X)^{0}$, wobei $\pi_{1}^{\mathrm{ab}}(X)^{0}$ den Kern von $\pi_{1}^{\mathrm{ab}}(X) \rightarrow \operatorname{Gal}\left(\bar{F} / \mathbb{F}_{q}\right)$ bezeichnet.

Verzweigung wird in einem Folgeartikel mittels höherer algebraischer $K$-Theorie (die wir nicht im Detail besprechen) behandelt.

Satz 6 (Kato-Saito (1986)). Sei U eine glatte, geometrisch irreduzible Varietät von Dimension d über $\mathbb{F}_{q}$ und $X$ eine normale projektive Varietät, die $U$ als dichte offene Teilmenge enthält. Dann gibt es eine exakte Sequenz

$$
0 \rightarrow \lim _{Z \subset X \backslash U} H_{\mathrm{Nis}}^{d}\left(X, \mathcal{K}_{d}(X, Z)\right) \rightarrow \pi_{1}^{\mathrm{ab}}(U) \rightarrow \hat{\mathbb{Z}} / \mathbb{Z} \rightarrow 0
$$

wobei der Limes über alle abgeschlossenen Unterschemata $Z$ in $X$ mit Träger in $X \backslash U$ zu bilden ist.

Hier bezeichnet $\mathcal{K}_{d}(X, Z)$ die relative höhere $K$-Garbe. Sie ist die der Prägarbe von relativen höheren $K$-Gruppen $V \mapsto K_{d}\left(V, V \times_{X} Z\right)$ zugeordnete Garbe. Die Idee, die diese beiden Resultate verbindet, ist Bloch's Formel, bewiesen von Quillen [31]. Angenommen, dass die normale Kompaktifizierung $X$ glatt ist, besagt Bloch's Formel für $X$, dass $\mathrm{CH}_{0}(X) \cong H_{\mathrm{Nis}}^{d}\left(X, \mathcal{K}_{d}\right)$, und nach dem ersten Satz von KatoSaito ist $\mathrm{CH}_{0}(X)$, bis auf Vervollständigung bezüglich des Grads, isomorph zu $\pi_{1}^{\mathrm{ab}}(X)$, die unverzweigte abelsche Fundamentalgruppe von $U$. Die Gruppen $H_{\mathrm{Nis}}^{d}\left(X, \mathcal{K}_{d}(X, Z)\right)$ tragen also zu demjenigen Teil von $\pi_{1}^{\mathrm{ab}}(U)$ bei, der möglicher Verzweigung entlang $X \backslash U$ zugeordnet ist.

\section{$4 \quad$ Kerz' Beiträge zur geometrischen Klassenkörpertheorie}

Da die höheren $K$-Garben eher schwer zu verstehen sind, wäre es vorteilhaft, eine Beschreibung von $\pi_{1}^{\mathrm{ab}}(U)$ zur Hand zu haben, die mehr dem unverzweigten Fall ähnelt. Dabei würde Verzweigung durch eine geometrischere Konstruktion erkannt, so wie im Fall von Kurven mithilfe von Moduln immer feinere Äquivalenzrelationen auf $Z_{0}(U)$ konstruiert werden, die immer höherer Verzweigung entsprechen. Solche Beschreibungen finden sich in wachsender Allgemeinheit in Arbeiten von Schmidt-Spieß, Kerz-Schmidt, KerzEsnault und Kerz.

In positiver Charakteristik $p$ ist zahme Verzweigung am einfachsten $\mathrm{zu}$ verstehen und kontrollieren. Ein Morphismus $f: C^{\prime} \rightarrow C$ zwischen glatten Kurven in positiver Charakteristik $p$ heißt zahm verzweigt in einem Punkt $q \in C^{\prime}$, falls, mit einem lokalen Parameter $t$ in $q$ ausgedrückt, $f$ die Form $f(t)=a \cdot t^{e}+$ höhere Terme annimmt, wobei $a \neq 0$ und $e$ teilerfremd zu $p$ ist. Es gibt viele Wege, diesen Begriff für höhere Dimensionen zu verallgemeinern. Falls allerdings eine glatte $\mathbb{F}_{q}$-Varietät $U$ eine projektive glatte Kompaktifizierung $X$ hat, so ist der abelianisierte zahme Quotient $\pi_{1}^{\mathrm{ab}}(U)^{t}$ von $\pi_{1}^{\mathrm{ab}}(U)$ einfach der zu $p$ teilerfremde Teil von $\pi_{1}^{\mathrm{ab}}(U)$ plus die $p^{\infty}$-Torsion in $\pi_{1}^{\mathrm{ab}}(X)$. Unter der Annahme, dass unsere glatte, geometrisch irreduzible Varietät $U$ über $\mathbb{F}_{q}$ eine glatte Kompaktifizierung $X$ hat, zeigen Schmidt und Spieß den folgenden Satz.

Satz 7 (Schmidt-Spieß (200o)). Sei $H_{0}^{\text {Sus }}(U)$ die 0-te Suslinhomologie von U. Dann definieren die Frobeniusabbildungen eine injektive Reziprozitätsabbildung $\operatorname{rec}_{U}: H_{0}^{\mathrm{Sus}}(U) \rightarrow \pi_{1}^{\mathrm{ab}}(U)^{t}$ mit Kokern $\hat{\mathbb{Z}} / \mathbb{Z}$.

Die 0-te Suslinhomologie $H_{0}^{\text {Sus }}(X)$ wird ähnlich wie $\mathrm{CH}_{0}(X)$ als Quotient von $Z_{0}(X)$ definiert, allerdings mittels einer etwas feineren Äquivalenzrelation. Sei $C \subset U \times \mathbb{P}^{1} \backslash\{1\}$ eine irreduzible Kurve, die über $\mathbb{P}^{1} \backslash\{1\} \cong \mathbb{A}^{1}$ endlich ist. Mittels der Kompaktifizierung $X$ von $U$ bedeutet das, dass $C \subset X \times \mathbb{P}^{1} \backslash\{1\}$ immer noch abgeschlossen und die Projektion auf $\mathbb{P}^{1} \backslash\{1\}$ nicht nur ein einzelner Punkt ist. Die Projektion $p_{2}$ ist dann einfach eine rationale Funktion auf $C$ und liefert ein Element $p_{1 *}\left(\operatorname{div}\left(p_{2}\right)\right) \in Z_{0}(U)$. Ist $R_{0}^{\text {Sus }}(U) \subset Z_{0}(U)$ die von solchen Elementen erzeugte Untergruppe, so definiert man $H_{0}^{\text {Sus }}(U)$ als den Quotienten

$$
H_{0}^{\text {Sus }}(U)=Z_{0}(U) / R_{0}^{\text {Sus }}(U) \text {. }
$$

Ist $p_{1}: C \rightarrow p_{1}(C)$ ein Isomorphismus, so ist der Divisor der induzierten Funktion $p_{2} \circ p_{1}^{-1}$ auf $p_{1}(C) \subset U$ offenbar ein Element von $Z_{0}(U)$, das in $\mathrm{CH}_{0}(U)$ auf Null abgebildet wird. Ein Standardargument zeigt dann, dass das weiterhin gilt, falls $C \rightarrow p_{1}(C)$ kein Isomorphismus ist. Folglich haben wir eine Surjektion $H_{0}^{\text {Sus }}(X) \rightarrow \mathrm{CH}_{0}(X)$. Allerdings ist die Bedingung, dass $p_{2}: C \rightarrow \mathbb{P}^{1} \backslash\{1\}$ endlich ist, eine zusätzliche Bedingung an die Funktion $p_{2}$. Ist $\bar{C}$ die projektive Vervollständigung von $C$, so besagt diese Bedingung, dass die rationale Funktion $p_{2}$ auf $\bar{C}$ auf allen Punkten von $\bar{C} \backslash C$ den Wert 1 annimmt. Anders gesagt haben wir eine Modulbedingung auf allen $C$ eingeführt, gewisserweise gleichmäßig über alle Kurven, die nach $U$ abbilden.

Nehmen wir der Einfachheit halber an, dass $\bar{C}$ glatt ist, so entspricht die Modulbedingung $f(p)=1$ für alle $p \in \bar{C} \backslash C$ entlang der Rosenlicht-Lang Beschreibung der Klassenkörpertheorie von $C$ genau den abelschen Überlagerungen $\bar{C}^{\prime} \rightarrow \bar{C}$, die über $C$ unverzweigt und über $\bar{C} \backslash C$ zahm verzweigt sind. Der Satz von Schmidt-Spieß besagt dann, dass die Relationen, die Suslinhomologie definieren, genau diejenigen sind, die diese zahme Verzweigungsbedingung entlang Kurven benutzen, um die abelschen Überlagerungen von $U$ auszuwählen, die entlang $X \backslash U$ zahm verzweigt sind.

Kerz und Schmid [13] überarbeiten einen Ansatz zur Klassenkörpertheorie arithmetischer Schemata, der ursprünglich auf Wiesend [37] zurückgeht und höhere algebraische $K$-Theorie, wie sie von Kato-Saito benutzt wird, vermeidet. Ohne Details zu nennen, liefert Wiesends Konstruktion eine Gruppe, die mittels der abgeschlossenen Punkte von $U$ und den Kurven auf $U$ definiert wird, als Verallgemeinerung $\mathcal{C}_{U}$ der klassischen Idealklassengruppe. Sie ist eine (nicht Hausdorffsche) topologische Gruppe mit einer stetigen Reziprozitätsabbildung $\operatorname{rec}_{U}: \mathcal{C}_{U} \rightarrow \pi_{1}(U)^{\mathrm{ab}}$, und unter 
einigen technischen Annahmen zeigen Kerz und Schmidt, dass der Kern von $\operatorname{rec}_{U}$ der Abschluss von 0 in $\mathcal{C}_{U}$ und der Kokern wie erwartet $\hat{\mathbb{Z}} / \mathbb{Z}$ ist. Wir erwähnen diesen Artikel, weil die Ideen, die von Wiesend und in [13] entwickelt wurden, einen Anfangspunkt für die geometrische Beschreibung des Kerns der Reziprozitätsabbildung bieten, wie sie von Kerz-Saito erreicht wurde und nachfolgend beschrieben wird.

Ein Hauch dieser geometrischen Beschreibung erscheint am Ende des Artikels [3] von Esnault und Kerz. Dort liegt der Schwerpunkt, einer Skizze Delignes folgend, auf einem Studium der Darstellungen mit beschränkter Verzweigung der étalen Fundamentalgruppe einer glatten Varietät $U$ über $\mathbb{F}_{q}$. Die Idee ist wieder sich auf Kurven, die nach $U$ abbilden, zu konzentrieren, und dabei eine feste Verzweigungsbedingung auf $U$ zu benutzen, um eine „gleichmäBige" Verzweigungsbedingung auf allen Kurven, die nach $U$ abbilden, festzusetzen. Ohne in die Details des Artikels einzugehen, würde ich gerne ein Resultat am Ende von [3] erwähnen. Für eine geeignete Kompaktifizierung $X$ von $U$ und einen effektiven Cartierdivisor $D$ auf $X$ mit Träger in $X \backslash U$, bieten Esnault-Kerz eine Definition ${ }^{4}$ einer Gruppe $\mathrm{CH}_{0}(X, D)$ von 0 -Zykeln mit Modul $D$ und zeigen, dass die Untergruppe von $\mathrm{CH}_{0}(X, D)$ bestehend aus Zykeln von Grad 0 endlich ist. Letzteres folgt aus Klassenkörpertheorie für $U$ und Delignes Endlichkeitssatz, dem Hauptresultat in [3]. Obwohl Esnault-Kerz einige frühere Quellen für ihre Definition von $\mathrm{CH}_{0}(X, D)$ nennen, erscheint mir die Definition in [3] das erste Aufkommen einer Verallgemeinerung der Gruppen $\mathrm{CH}_{0}(C, \mathfrak{m})$ von Kurven zu höherdimensionalen Varietäten zu sein. Die Definition von Esnault-Kerz wurde von Kerz-Saito in [9] leicht verändert durch eine unbedeutende Reindizierung und BindaKrishna [16] haben die Kerz-Saito Version aus einigen früheren Konstruktionen, die von Esnault-Kerz erwähnt wurden, wiedergewonnen, aber die Esnault-Kerz/Kerz-Saito Definition hat den Vorteil, dass sie im Gegensatz zu früheren Konstruktionen offensichtlich vom Divisor $D$ abhängt.

Nun hier die Kerz-Saito Definition von $\mathrm{CH}_{0}(X, D)$. Sei wie oben $U$ eine glatte Varietät über $\mathbb{F}_{q}$ und $X$ eine normale, eigentliche Varietät, die $U$ als dichte offene Teilmenge enthält, sodass das Komplement $X \backslash U$ der Träger eines effektiven Cartierdivisors ist. Sei weiter $D$ ein effektiver Cartierdivisor mit Träger in $X \backslash U$. Wir betrachten eine glatte irreduzible projektive Kurve $C$ über $\mathbb{F}_{q}$ mit einer Abbildung $p: C \rightarrow X$, für die $p(C)$ nicht in $X \backslash U$ enthalten ist, und einer rationalen Funktion $f$ auf $C$, die folgende Bedingungen erfülllt.

(i) Die Funktion $f$ ist regulär an allen Punkten $x \in C$ mit $p(x) \in X \backslash U$.

(ii) Schreiben wir $p^{*}(D)=\sum_{i=1}^{r} n_{i} x_{i}$, so ist $\operatorname{ord}_{x_{i}} f-1 \geq n_{i}$ für alle $i$.

Sei $R_{0}(X, D) \subset Z_{0}(U)$ die Untergruppe, die von $p_{*}(\operatorname{div}(f))$ für $p: C \rightarrow X$ und $f$ wie oben erzeugt wird, und setze $\mathrm{CH}_{0}(X, D):=Z_{0}(U) / R_{0}(X, D)$. Anders gesagt ist $\mathrm{CH}_{0}(X, D)$ der Quotient von $Z_{0}(U)$ modulo der Untergruppe von 0Zykeln $p_{*}(z)$ für $p: C \rightarrow X$ wie oben und $z \in Z_{0}\left(p^{-1}(U)\right)$, sodass $z$ in $\mathrm{CH}_{0}\left(C, p^{*}(D)\right)$ auf Null abgebildet wird.
Nehmen wir den Limes der $\mathrm{CH}_{0}(X, D)$ über immer gröBere $D$, erhalten wir die topologische Gruppe $C(U)$. Wie zuvor steigen die Frobeniusabbildungen für Punkte $x \in U$ zu einer Reziprozitätsabbildung

$$
\operatorname{rec}_{U}: C(U) \rightarrow \pi_{1}^{\mathrm{ab}}(U)
$$

ab. Führen wir dieselbe Konstruktion für die Untergruppe $\mathrm{CH}_{0}(X, D)_{0} \subset \mathrm{CH}_{0}(X, D)$ der Zykeln von Grad 0 aus, erhalten wir die abgeschlossene Untergruppe $C(U)_{0} \subset C(U)$ und die Reziprozitätsabbildung

$$
\operatorname{rec}_{U}^{0}: C(U)_{)} \rightarrow \pi_{1}^{\mathrm{e} t}(U)_{0}^{\mathrm{ab}},
$$

sowie im Endlichen Abbildungen

$$
\begin{aligned}
& \operatorname{rec}_{X, D}: \mathrm{CH}_{0}(X, D) \rightarrow \pi_{1}^{\mathrm{ab}}(X, D) ; \\
& \operatorname{rec}_{X, D}^{0}: \mathrm{CH}_{0}(X, D)^{0} \rightarrow \pi_{1}^{\mathrm{ab}}(X, D)^{0} .
\end{aligned}
$$

Hier ist $\pi_{1}^{\mathrm{ab}}(X, D)$ die Galoisgruppe der maximalen unverzweigten abelschen Überlagerung von $U$, mit Verzweigung beschränkt durch $D$, und $\pi_{1}^{\mathrm{ab}}(X, D)^{0}$ die ähnlich definierte Untergruppe von $\pi_{1}^{\mathrm{ab}}(X, D)$ für Überlagerungen von $U \times \mathbb{F}_{q} \overline{\mathbb{F}}$.

Die relativen 0-Zykel nach Kerz-Saito erlauben eine Beschreibung der Klassenkörpertheorie für $U$, die exakt parallel zu der von Kurven verläuft.

Satz 8 (Kerz-Saito). Sei char $\mathbb{F}_{q} \neq 2$. Dann ist die Abbildung $\operatorname{rec}_{U}^{0}$ ein topologischer Isomorphismus. Für jedes $D$ ist $\operatorname{rec}_{X, D}^{0}$ ein Isomorphismus zwischen endlichen Gruppen.

Obwohl es nicht so in Kerz-Saito erwähnt wird, zeigt dieses Ergebnis, dass $\operatorname{rec}_{U}$ und $\operatorname{rec}_{X, D}$ beide injektiv mit Kokern $\hat{\mathbb{Z}} / \mathbb{Z}$ sind. Eine Konsequenz aus dem Satz von KerzSaito ist ein neuer Beweis der Endlichkeit von $\mathrm{CH}_{0}(X, D)^{0}$. Dieses Resultat bejaht auch eine Frage zu Darstellungen von $\pi_{1}^{\text {ét }}(U)$, die von Deligne gestellt und in [3] diskutiert wurde. Wir stellen sie hier etwas unpräzise vor: gegeben eine Familie von $l$-adischen Galoisdarstellungen $\rho_{C}$ für jede Kurve $C \subset U$, mit Verträglichkeitsbedingungen auf den Schnitten in $U$ und mit gleichmäßig durch einen Cartierdivisor $D$ auf $X$ beschränkter Verzweigung, ein sogenanntes Garbenskelett, gibt es eine entsprechende Darstellung von $\pi_{1}^{\text {ét }}(U)$ mit ähnlich beschränkter Verzweigung? Rang Eins führt zu Darstellungen von $\pi_{1}^{\mathrm{ab}}(U)$, und die Beschreibung von $\pi_{1}^{\mathrm{ab}}(X, D)^{0}$ nach Kerz-Saito erlaubt Delignes Frage in diesem Fall zu bejahen (falls die Charakteristik nicht 2 ist). ${ }^{5}$

Die relative Chowgruppe nach Kerz-Saito passt in eine umfassendere Entwicklung höherer Chowgruppen mit Mo$d u l$, beginnend bei Bloch-Esnault [18] in ihrer Konstruktion additiver Chowgruppen. Während diese Theorie ursprünglich als Mittel zum Verständnis der Deformationstheorie von Chowgruppen eingeführt wurde, ist sie mittlerweile so weit ausgereift, dass sie kategorifiziert werden konnte im Versuch, Voevodskys triangulierte Kategorie von Motiven zu verfeinern, um Anwendungen auf eine größere Breite von Phänomenen zu erlauben, einschließlich der vorgehend beschriebenen wilden Verzweigung, aber auch auf Stokesphänomene und irreguläre Zusammenhänge, de Rham-WittKomplexe und viele andere Beispiele. Aus den Anwendungen auf die Klassenkörpertheorie entwickeln Kahn, Ivorra, 
Rülling, Saito und Yamazaki $[20,22]$ eine Theorie von „Reziprozitätsgarben“, die Voevodskys Theorie von homotopieinvarianten Garben mit Transfer verallgemeinert. Arbeiten von Park, Krishna, Kai und Iwasa $[21,23,26]$ haben die Theorie von 0-Zykeln mit Modul zu einer sinnvollen Theorie von höheren Chowgruppen mit Modul ausgeweitet. All dies um zu sagen, dass Kerz' Arbeit auf diesem Gebiet breite Resonanz gefunden und zu Entwicklungen in vielen neuen Richtungen geführt hat.

\section{Anmerkungen}

1. „Eigentlich“ ist die algebraische Version von kompakt.

2. Nicht zu verwechseln mit einem Modul über einem Ring, siehe [29, III, §7, Seite 181]

3. Grob gesagt heißt das, dass $X$ eine endliche Vereinigung von Teilen ist, die jeweils durch endlich viele Polynomgleichungen mit ganzzahligen Koeffizienten definiert sind.

4. Unsere Notation unterscheidet sich leicht von der in [3] und folgt stattdessen [9].

5. Die Diskussion von Kerz-Saito wurde von Binda-Krishna-Saito überarbeitet, und diese neuen Resultate decken auch den Fall von Charakteristik $2 \mathrm{ab}$.

\section{Übersetzung aus dem Englischen von Viktor Kleen}

\section{Literatur}

[1] Bloch, Spencer; Esnault, Hélène; Kerz, Moritz, Deformation of algebraic cycle classes in characteristic zero. Algebr. Geom. 1 (2014), no. 3, 290-310.

[2] Bloch, Spencer; Esnault, Hélène; Kerz, Moritz, p-adic deformation of algebraic cycle classes. Invent. Math. 195 (2014), no. 3, 673-722.

[3] Esnault, Hélène; Kerz, Moritz, A finiteness theorem for Galois representations of function fields over finite fields (after Deligne). Acta Math. Vietnam. 37 (2012), no. 4, 531-562.

[4] Esnault, Hélène; Kerz, Moritz; Wittenberg, Olivier, A restriction isomorphism for cycles of relative dimension zero. Camb. J. Math. 4 (2016), no. 2, 163-196.

[5] Kerz, Moritz, On negative algebraic K-groups. Proceedings of the International Congress of Mathematicians - Rio de Janeiro 2018. Vol. II. Invited lectures, 163-172, World Sci. Publ., Hackensack, NJ, 2018.

[6] Kerz, Moritz, Milnor K-theory of local rings with finite residue fields. J. Algebraic Geom. 19 (2010), no. 1, 173-191.

[7] Kerz, Moritz, The Gersten conjecture for Milnor K-theory. Invent. Math. 175 (2009), no. 1, 1-33

[8] Kerz, Moritz; Müller-Stach, Stefan The Milnor-Chow homomorphism revisited. $K$-Theory 38 (2007), no. 1, 49-58.

[9] Kerz, Moritz; Saito, Shuji, Chow group of o-cycles with modulus and higher-dimensional class field theory. Duke Math. J. 165 (2016), no. 15, 2811-2897.

[10] Kerz, Moritz; Saito, Shuji, Cohomological Hasse principle and resolution of quotient singularities. New York J. Math. 19 (2013), 597-645

[11] Kerz, Moritz; Saito, Shuji, Cohomological Hasse principle and motivic cohomology for arithmetic schemes. Publ. Math. Inst. Hautes Études Sci. 115 (2012), 123-183.

[12] Kerz, Moritz; Schmidt, Alexander, On different notions of tameness in arithmetic geometry. Math. Ann. 346 (2010), no. 3, 641-668.

[13] Kerz, Moritz; Schmidt, Alexander, Covering data and higher dimensional global class field theory. J Number Theory 129 (2009), no 10, 2569-2599.
[14] Kerz, Moritz; Strunk, Florian, On the vanishing of negative homotopy K-theory. J. Pure Appl. Algebra 221 (2017), no. 7, 1641-1644.

[15] Kerz, Moritz; Strunk, Florian; Tamme, Georg, Algebraic K-theory and descent for blow-ups. Invent. Math. 211 (2018), no. 2, 523-577.

\section{Ergänzende Literatur}

[16] Binda, Federico; Krishna, Amalendu, Zero cycles with modulus and zero cycles on singular varieties. Compos. Math. 154 (2018), no. 1, 120-187.

[17] Federico Binda, Amalendu Krishna, Shuji Saito, Bloch's formula for o-cycles with modulus and higher dimensional Class Field Theory. arXiv:2002.01856 [math.AG]

[18] Bloch, Spencer; Esnault, Hélène, An additive version of higher Chow groups. Ann. Sci. École Norm. Sup. (4) 36 (2003), no. 3, 463-477.

[19] Harbater, David, Fundamental groups and embedding problems in characteristic $p$. Recent developments in the inverse Galois problem (Seattle, WA, 1993), 353-369, Contemp. Math., 186, Amer. Math. Soc., Providence, RI, 1995.

[20] Ivorra, Florian; Rülling, Kay, K-groups of reciprocity functors. J. Algebraic Geom. 26 (2017), no. 2, 199-278.

[21] Iwasa, Ryomei; Kai, Wataru; Chern classes with modulus. Nagoya Math. J. 236 (2019), 84-133.

[22] Kahn, Bruno; Saito, Shuji; Yamazaki, Takao, Reciprocity sheaves. With two appendices by Kay Rülling. Compos. Math. 152 (2016), no. $9,1851-1898$.

[23] Kai, Wataru, Chow's moving lemma with modulus. K-Theory - Proceedings of the International Colloquium, Mumbai, 2016, 127142, Hindustan Book Agency, New Delhi, 2018.

[24] K. Kato, S. Saito, Unramified class field theory of arithmetical surfaces, Ann. of Math. (2) 118 (2) (1983) 241-275.

[25] K. Kato, S. Saito, Global class field theory of arithmetic schemes, in: Applications of Algebraic K-Theory to Algebraic Geometry and Number Theory, Parts I, II, Boulder, CO, 1983, in: Contemp. Math., vol. 55, Amer. Math. Soc., Providence, RI, 1986, pp. 255-331.

[26] Krishna, Amalendu; Park, Jinhyun, On additive higher Chow groups of affine schemes. Doc. Math. 21 (2016), 49-89.

[27] Lang, Serge, Sur les s'eries L d'une variété alg'ebrique. Bull. Soc. Math. France 84 (1956), 385-407.

[28] Lang, Serge, Unramified class field theory over function fields in several variables. Ann. of Math. (2) 64 (1956), 285-325.

[29] Neukirch, Jürgen Klassenkörpertheorie. Newly edited and with a foreword by Alexander Schmidt. Springer-Lehrbuch. Springer, Heidelberg, 2011.

[30] Pop, Florian Étale Galois covers of affine smooth curves. The geometric case of a conjecture of Shafarevich. On Abhyankar's conjecture. Invent. Math. 120 (1995), no. 3, 555-578.

[31] Quillen, Daniel, Higher algebraic K-theory. I. Algebraic K-theory, I: Higher K-theories (Proc. Conf., Battelle Memorial Inst., Seattle, Wash., 1972), pp. 85-147. Lecture Notes in Math., Vol. 341, Springer, Berlin 1973.

[32] Roquette, Peter, Class field theory in characteristic $p$, its origin and development. Class field theory - its centenary and prospect (Tokyo, 1998), 549-631, Adv. Stud. Pure Math., 30, Math. Soc. Japan, Tokyo, 2001.

[33] Rosenlicht, Maxwell, A universal mapping property of generalized jacobian varieties. Ann. of Math. (2) 66 (1957), 8o-88.

[34] Rosenlicht, Maxwell, Generalized Jacobian varieties. Ann. of Math. (2) 59 (1954), 505-530.

[35] Schmidt, Alexander; Spieß, Michael, Singular homology and class field theory of varieties over finite fields. J. Reine Angew. Math. 527 (2000), 13-36.

[36] Serre, Jean-Pierre, Groupes alg'ebriques et corps de classes. Publications de l'institut de math'ematique de l'universit'e de Nancago, VII. Hermann, Paris 1959

[37] Wiesend, Götz, Class field theory for arithmetic schemes. Math. Z. 256 (2007), no. 4, 717-729.

Prof. Dr. Marc Levine, Fakultät Mathematik, Universität Duisburg-Essen,

Campus Essen, Thea-Leymann-Straße 9, 45127 Essen

marc.levine@uni-due.de

Marc Levine hat am MIT studiert und 1979 an der Brandeis University bei Teruhisa Matsusaka promoviert. Seit 2009 lehrt er an der Universität Duisburg-Essen, bis 2014 auf einer Humboldt-Professur. Forschungsschwerpunkte: Algebraische Geometrie, Topologie, Algebra, Theorie des algebraischen Kobordismus, motivische Kohomologie, algebraische K-Theorie. 